Normalisation of real-time RT-PCR gene expression measurements in Arabidopsis thaliana exposed to increased metal concentrations Non Peer-reviewed author version

REMANS, Tony; SMEETS, Karen; OPDENAKKER, Kelly; MATHIJSEN, Dennis; VANGRONSVELD, Jaco \& CUYPERS, Ann (2008) Normalisation of real-time RT-PCR gene expression measurements in Arabidopsis thaliana exposed to increased metal concentrations. In: PLANTA, 227(6). p. 1343-1349.

DOI: $10.1007 / \mathrm{s} 00425-008-0706-4$

Handle: http://hdl.handle.net/1942/8264 


\title{
Normalisation of real-time RT-PCR gene expression measurements in Arabidopsis thaliana exposed to increased metal concentrations
}

Tony Remans, Karen Smeets, Kelly Opdenakker, Dennis Mathijsen, Jaco Vangronsveld, Ann Cuypers

Environmental Biology, Hasselt University, Agoralaan D, 3590 Diepenbeek, Belgium

Corresponding author: Tony Remans (tony.remans@uhasselt.be)

\begin{abstract}
Accurate quantification by real-time RT-PCR relies on normalisation of the measured gene expression data. Normalisation with multiple reference genes is becoming the standard, but the best reference genes for gene expression studies within one organism may depend on the applied treatments or the organs and tissues studied. Ideally, reference genes should be evaluated in all experimental systems. A number of candidate reference genes for Arabidopsis have been proposed, which can be used as a starting point to evaluate their expression stability in individual experimental systems by available computer algorithms like geNorm and NormFinder. Using this approach, we identified the best three reference genes from a set of ten candidates - that included three traditional "housekeeping” genes - for normalisation of gene expression when roots and leaves of Arabidopsis thaliana are exposed to cadmium (Cd) and copper (Cu). The expression stabilities of AT5G15710 (F-box protein), AT2G28390 (SAND family protein), and AT5G08290 (mitosis protein YLS8) were the highest when considering the effect to the roots and shoots of $\mathrm{Cd}$ and $\mathrm{Cu}$ treatments. Even though the effect of $\mathrm{Cd}$ and excess $\mathrm{Cu}$ on the plants is very different, the same best reference genes were identified when considering $\mathrm{Cd}$ or $\mathrm{Cu}$ treatments separately. This suggests that these three genes may also be suitable when studying the gene expression after exposure of Arabidopsis thaliana to increased concentrations of other metals.
\end{abstract}

\section{Keywords}

Cadmium, Copper, Metal stress, Reference genes, Housekeeping genes, Real-time PCR, qBase, geNorm, NormFinder 


\section{Abbreviations}

Cd, Cadmium; $\mathrm{Cu}$, Copper; ROS, Reactive oxygen species 


\section{Introduction}

Plants need essential micro-elements to grow. Some of these are metals that can become phytotoxic at higher concentrations (e.g. copper, $\mathrm{Cu}$ ). Other metals are non-essential trace elements that are toxic for plants at certain exposure levels (e.g. cadmium, Cd). Metal toxicity can cause a disruption of physiological processes, such as photosynthesis and transpiration, which can lead to chlorosis, necrosis and reduced growth (Baryla et al. 2001; Prasad and Zeeshan, 2005). An underlying effect of increased exposure to metals is oxidative stress (Cuypers et al. 2000; Cuypers et al. 2002; Ortega-Villasante et al. 2005; Smeets et al. 2005; Rodriguez-Serrano et al. 2006), which occurs when the balance between oxidants (such as reactive oxygen species, ROS), and antioxidants (which include certain enzymes and metabolites), is shifted towards the oxidants. Elevated levels of ROS can cause cellular damage, but are also important in stress signalling (Mittler et al. 2004). Multiple authors have demonstrated responses at the biochemical-physiological level and on signal transduction pathways induced by exposure to relatively high (acute) metal concentrations in plants (Jonak et al. 2004; Maksymiec and Krupa, 2006, Yeh et al. 2007), whereas in our studies, we expose Arabidopsis thaliana to lower sub-lethal concentrations of $\mathrm{Cu}$ or $\mathrm{Cd}$. At these low exposures to metals no visible changes to the plant can be detected, but a number of changes are taking place at the cellular level.

For measuring immediate molecular responses, a reliable quantification of gene expression is important and of major importance herein is the normalisation of the measured expression levels for the genes of interest. This is necessary to correct for variability that can arise and accumulate at several steps and is related to the quality of the RNA samples, the RNA input in the reverse transcription reaction, the enzymatic efficiencies in the reverse transcription reaction, and differences between tissues, cells and treatments in overall transcriptional activity. For whole plant samples, normalisation against the number of cells is not possible and we also like to avoid normalisation against the total RNA mass included in the reverse transcription reaction, because total RNA is not representative for the mRNA fraction (Vandesompele et al. 2002). Mostly, normalisation of gene expression is achieved by measuring endogenous control genes. Expression of these genes should not vary in the tissues investigated or in function of the treatment, something which ideally should be verified in every single experimental set-up.

Reference genes that have been used until now are "housekeeping genes”, which are involved in basic cellular processes, and they were supposed to have a uniform level of expression 
across different treatments, organs and developmental stages. Previous reports in which gene expression is measured either quantitatively or semi-quantitatively in response to heavy metal stress, mostly make use of EF-1 $\alpha$ (At5g60390) or ACT2 (At5g09810) genes (Bovet et al. 2003; Becher et al. 2004; Cagnac et al. 2004; Herbette et al. 2006; Talke et al. 2006; Weber et al. 2006; Sunkar et al. 2006). However, the expression of traditional "housekeeping genes” is not always stable (Thellin et al. 1999; Czechowski et al. 2005). Furthermore, normalisation to a single endogenous gene can lead to erroneous normalisation (Vandesompele et al. 2002).

Many publications exist in animal and human research that describe the identification of multiple reference genes for normalisation of real-time RT-PCR data, but similar reports are extremely rare in plant research. Traditional "housekeeping genes” were evaluated for studies of seagrass subjected to heat stress (Ransbotyn and Reusch 2006); for rice plants under hormone, salt and drought treatment and in different developmental stages (Jain et al. 2006); for potato under different biotic and abiotic stresses (Nicot et al. 2005); and for poplar in different tissues (Brunner et al. 2004). Czechowski et al. (2005) moved away from the typical "housekeeping” genes and identified a new set of Arabidopsis thaliana genes, some of which with unknown function, from experiments using Affymetrix ATH1 probe sets related to development, abiotic stress, biotic stress, hormones, nutrient stress and light treatments. The expression stability of 20 novel reference genes derived from that analysis and of 5 traditional housekeeping genes was verified by real-time RT-PCR on cDNA samples from ecotype Columbia-0 grown under various conditions and stress treatments and from different organs. The abiotic stress treatments in particular included salt $(\mathrm{NaCl})$, drought (mannitol), nutrient deprivation (sulphur, carbohydrate, phosphorus) and cold treatment. Since exposure to increased concentrations of metals was not included in that study, we selected the 10 best reference genes together with some traditional housekeeping genes and evaluated their expression stability in our experimental system using the available algorithms geNorm (Vandesompele et al. 2002) and NormFinder (Andersen et al. 2004). These algorithms evaluate the relative expression stability of multiple candidate reference genes and have been developed to address the circular problem that for the evaluation of the expression stability of a candidate reference gene another one is needed for its normalisation. We propose that validation of selected candidate reference genes from Czechowski et al. (2005) using such an algorithm is straightforward and should be the basis of any gene expression study in Arabidopsis thaliana. Here, using this approach, we identified the best reference genes after exposure of Arabidopsis thaliana to $\mathrm{Cd}$ and $\mathrm{Cu}$, and the results suggest that these genes may also be suitable candidates when studying exposure to other metals. 


\section{Materials and methods}

Plant CUlture AND HaRvest. Arabidopsis thaliana wild-type plants of the ecotype Columbia (Col-0; Lehle seeds, Round Rock, Texas, USA) were grown in hydroponic culture (Smeets et al. 2007) under a $12 \mathrm{~h} / 22^{\circ} \mathrm{C}$ day and $12 \mathrm{~h} / 18^{\circ}$ night regime and a photosynthetic photon flux density of $150 \mu \mathrm{mol} \mathrm{m}{ }^{-2} \mathrm{~s}^{-1}$ at the leaf level. Three-week-old plants were exposed 1 hour into the photoperiod to relatively low $(2 \mu \mathrm{M})$ or high $(10 \mu \mathrm{M})$ concentration of $\mathrm{CdSO}_{4}$, or to relatively low $(0.5 \mu \mathrm{M})$ or high $(2 \mu \mathrm{M})$ concentration of $\mathrm{CuSO}_{4}$, whereas some plants were left untreated (control plants). For each treatment, 4 root samples and 4 leaf samples of 50-100 mg, each containing tissue originating from 3 to 8 plants, were taken $24 \mathrm{~h}$ after the start of treatment and snap frozen in liquid nitrogen before storage at $-70^{\circ} \mathrm{C}$.

RNA ISOLATION, QUALITY CONTROL AND CDNA SYNTHESIS. Frozen tissues in $2 \mathrm{ml}$ micocentrifuge tubes were disrupted under frozen conditions using two stainless steel beads (2mm diameter) in each sample and the Retsch Mixer Mill MM2000. RNA was extracted from the disrupted tissue using the RNeasy Plant Mini Kit (Qiagen). After measuring the RNA concentration on the NanoDrop ND-1000 spectrophotometer (NanoDrop Technologies), all RNA samples were adjusted to the same concentration, measured and adjusted again to homogenize RNA input in the subsequent cDNA synthesis reaction. RNA quality was assessed using the Agilent-2100 Bioanalyzer and RNA 6000 NanoChips (Agilent Technologies). One $\mu \mathrm{g}$ was used in a $20 \mu$ Q Quantitect Reverse Transcription reaction (Qiagen), which includes a genomic DNA elimination step and makes use of random hexamer priming. A tenfold dilution of the cDNA was made using 1/10 diluted TE buffer (1 mM Tris$\mathrm{HCl}, 0.1 \mathrm{mM}$ EDTA, $\mathrm{pH} 8.0$ ) and stored at $-20^{\circ} \mathrm{C}$.

REAL-TIME PCR AND DATA ANALYSIS. Primers sequences for the reference genes were taken from Czechowski et al. (2005) and are listed in Table 1. Real-Time PCR was performed in an optical 96-well plate with an ABI PRISM 7000 sequence detection system (Applied Biosystems) and universal cycling conditions $\left(10 \mathrm{~min} 95^{\circ} \mathrm{C}, 40\right.$ cycles of $15 \mathrm{~s}$ at $95^{\circ} \mathrm{C}$ and 60 $\mathrm{s}$ at $60^{\circ} \mathrm{C}$ ) followed by the generation of a dissociation curve to check for specificity of amplification. Reactions contained SYBR Green Master Mix (Applied Biosystems), $300 \mathrm{nM}$ of a gene specific forward and reverse primer, and $2.5 \mu \mathrm{l}$ of the diluted cDNA in each $25 \mu \mathrm{l}$ 
reaction. "No template controls" contained $2.5 \mu \mathrm{l}$ RNase free water instead. Primer efficiencies and standard deviations were calculated using qBase software v1.3.5 (Hellemans et al. 2007) on a standard curve generated using a 4-fold dilution series of one sample over at least 5 dilution points that were measured in triplicate. Expression levels for each sample were calculated on two technical replicates via the standard curve (which takes into account primer efficiency) and expressed relative to the sample with the highest expression before geNorm v3.4 (Vandesompele et al. 2002) or NormFinder (Andersen et al. 2004) input.

\section{Results}

The ten most stably expressed genes for Arabidopsis thaliana ecotype Col-0 as identified by Czechowski et al. (2005) and three traditional housekeeping genes EF-1a, ACT2 and UBQ10, were chosen for evaluation of expression stability during metal treatment. These candidate reference genes were amplified in cDNA samples from roots and from leaves, issued from 4 different exposures to increased metal concentrations ( $2 \mu \mathrm{M} \mathrm{Cd}, 10 \mu \mathrm{M} \mathrm{Cd}, 0.5 \mu \mathrm{M} \mathrm{Cu}$ and 2 $\mu \mathrm{M} \mathrm{Cu}$ ), and from one control treatment (not exposed to increased metal concentrations). These exposure concentrations were chosen because we found that they lead to effects on the molecular level, but still allow adaptation of the plants without any major apoptotic or necrotic effects. The concentrations of $\mathrm{Cu}$ that were applied are lower compared to $\mathrm{Cd}$, because the toxicity of this metal at similar concentrations is higher, most likely due to its redox-active properties (Fargasova 2001). Melting curve analysis of the amplification products confirmed that the primers amplified a single product (results not shown). A 4-fold dilution series of one of the samples was used to prepare a standard curve from which primer efficiency was calculated using the formula $E=10^{-1 / S L O P E}$ (Table 2). The primer pairs for AT2G32170, AT4G33380 and AT5G46630 resulted in an inefficient amplification and were therefore eliminated from further analyses. Average $\mathrm{Ct}$ values for the ten remaining candidate reference genes were in the range from 15 to 31 and the three traditional housekeeping genes EF1-a, ACT2 and UBQ10 were the most highly expressed, together with AT5G08290 (Table 2).

To be able to evaluate the effect of $\mathrm{Cu}$ and $\mathrm{Cd}$ treatment on gene expression in roots and leaves of Arabidopsis thaliana, we should normalise all samples that are to be compared by the same reference genes. We therefore used the relative expression values for each cDNA sample as input for the geNorm algorithm, from which a measure $\mathrm{M}$ is calculated for each 
reference gene. This measure $\mathrm{M}$ reflects the expression stability of the gene compared to the other reference genes - a lower M-value means more stable gene expression - and is the basis for the ranking of the genes in order of their expression stability. When considering all treatments and organs, AT5G15710 and AT2G28390 are the best reference genes, followed by AT5G08290 (Fig. 1a).

The next question is how many reference genes should be included for normalisation of gene expression. The geNorm algorithm also determines the pairwise variation $\mathrm{Vn} / \mathrm{n}+1$, which measures the effect of adding further reference genes on the normalisation factor (that is calculated as the geometric mean of the expression values of the selected reference genes). It is advisable to add additional reference genes to the normalisation factor until the added gene has no significant effect (low V-value). Vandesompele et al. (2002) used 0.15 as a cut-off value, below which the inclusion of an additional reference gene is not required, but this is not an absolute rule and depends on the data. The V-graph (Fig. 1d) shows that two reference genes are sufficient for normalisation, as V2/3 $<0.15$ and that inclusion of additional reference genes does not considerably decrease the $\mathrm{Vn} / \mathrm{n}+1$ values. However, Vandesompele et al. (2002) recommend the minimal use of three reference genes. Addition of a fourth reference gene $(E F-1 a)$ increases the pairwise variation, and we therefore considered three reference genes to be optimal for our experiments. The NormFinder algorithm also identifies the same three best reference genes as geNorm, although the ranking is slightly different (Supplemental Table 1a).

To evaluate the separate impact of the two metals on the ranking of the reference genes, we changed the geNorm input to consider only the Cd treatments (samples from Cd treated plants inclusive the control samples), or the $\mathrm{Cu}$ treatments (samples from $\mathrm{Cu}$ treated plants inclusive the control samples). Interestingly, the three best reference genes are the same ones for both, the Cd and Cu treatments (Fig. 1b,c). The ranking of these three genes for Cd treatment is the same as when all treatments were considered, but for the $\mathrm{Cu}$ treatment AT5G08290 is a slightly better reference gene than AT2G28390, although the difference in M-value is minor. The pairwise variation graph (Fig. 1b) again shows that 2 reference genes are sufficient, but the inclusion of a third reference gene is advisable. Also apparent is that over the whole curve, the $\mathrm{M}$-values of the reference genes are higher in $\mathrm{Cu}$ treatments than in $\mathrm{Cd}$ treatments (compare Fig. 1b and 1c), which indicates that expression of these genes is a little less stable under $\mathrm{Cu}$ exposure. The NormFinder algorithm identified for the Cd treatments the same best three reference genes in the same order as the geNorm algorithm (Supplemental Table 1b). 
For the $\mathrm{Cu}$ treatments, the best two reference genes were the same, but from then on there is a difference in ranking (Supplemental Table1c).

The traditional housekeeping genes included in this experiment (ACT2, EF-1a, UBQ10) are according to geNorm ranked immediately behind the three best reference genes (except for $U B Q 10$ during $\mathrm{Cu}$ treatments), and the ranking of the other genes too is very similar for the different treatments (Fig. 1a,b,c). In both geNorm and NormFinder, AT4G34270 was ranked as the worst candidate reference gene of the ten (Fig. 1a-c; Supplemental Table 2a-c). Supplemental Fig. 1 shows the expression stability and pairwise variation graphs of other data groups, for example, looking at leaves and roots separately. AT5G15710, AT2G28390 and AT5G08290 are still the best reference genes in most of these groups, except when evaluating the separate data sets related to the effect of $\mathrm{Cu}$ exposure in the leaves and $\mathrm{Cd}$ exposure in the roots.

In our geNorm input, we kept the biological replicates separated. However, in some previous publications that identified reference genes for plant research, biological replicates were grouped post-amplification by using relative expression data derived from an average $\mathrm{Ct}$ value for the biological replicates as input for geNorm (Jain et al. 2006) or pre-amplification by using cDNA pools as real-time PCR input (Czechowski et al. 2005). We entered data for each individual sample because this also takes into account any variation in expression between biological replicates that is not due to the treatments. Separate input of biological replicates is also needed for the calculation of a normalisation factor for each individual cDNA sample. We evaluated the impact of alternative geNorm input on the ranking of the reference genes by entering averaged values for each treatment/organ group into geNorm or NormFinder. The result is a change in the ranking of the genes, and the effect is especially apparent for AT4G34270, which shows a more stable expression according to both the geNorm (Supplemental Figure 2) and NormFinder (Supplemental Table 1) algorithms. According to geNorm it is even the best reference gene when looking at leaf samples of the Cd treatment data group (Supplemental Figure 2). We suspect that this apparently increased expression stability of AT4G34270 is due to the elimination of variation in expression between the biological replicates of a treatment/organ group when these were averaged for the alternative input. 


\section{Discussion}

Real-Time PCR is becoming more and more applied as a method for gene expression analysis. Reliable quantification of gene expression relies on trustworthy normalisation of real-time PCR data. The best normalisation is obtained using internal reference genes because it takes into account variation introduced by RNA sample quality, RNA input quantity and enzymatic efficiency in reverse transcription. Still, on the technical side, we believe it is important to homogenize these input parameters by accurately measuring RNA concentrations and assessing RNA sample quality. RNA sample concentrations were measured twice on the Nanodrop spectrophotometer, and the samples were adjusted to the same concentration between each measurement. As a result we can enter similar volumes of accurately quantified RNA in each reverse transcription reaction, thereby homogenizing RNA input quantity. Furthermore, testing the RNA samples using the Bioanalyzer ensures RNA quality, which has an influence on reverse transcription efficiency. When using SYBR green for real-time PCR quantification, genes of interest and internal control genes are measured in separate wells and usually even in separate plates. Therefore, accurate pipetting while entering the cDNA during real-time PCR reaction set-up is extremely important, as differences in cDNA input between gene of interest and reference genes will cause an erroneous normalisation by the reference gene.

The ideal reference genes have a constant level of expression that is not different in the organs or tissues studied, and that is not influenced by the applied treatments. Since a certain variation always exists for any reference gene, normalisation of gene expression with a single reference gene can bias the results. Normalisation with multiple reference genes is becoming the standard, but reports that identify such genes in plant research are limited, even though algorithms are available to test the expression stability of candidates (Andersen et al. 2004; Pfaffl et al. 2004; Vandesompele et al. 2002) and a number of candidate reference genes for Arabidopsis have been proposed (Czechowski et al. 2005). To obtain a solid basis for normalisation of our gene expression data when studying the effects of $\mathrm{Cd}$ and $\mathrm{Cu}$ exposure in roots and leaves of Arabidopsis thaliana, we evaluated the expression stability of 10 candidate reference genes, including three traditional "housekeeping” genes. AT5G15710 (Fbox protein), AT2G28390 (SAND family protein), and AT5G08290 (mitosis protein YLS8) were identified in our experiment as the three best candidates for normalisation with multiple reference genes by both geNorm and NormFinder. We also looked at the $\mathrm{Cd}$ and $\mathrm{Cu}$ exposure separately, and identified the same three best reference genes, even though the expression 
stability of the candidate reference genes is generally lower in $\mathrm{Cu}$ treatments than in $\mathrm{Cd}$ treatments. The traditional housekeeping genes included in this experiment (ACT2, EF-1a, $U B Q 10$ ) are according to geNorm ranked immediately behind the three best reference genes, although with NormFinder they are ranked lower. Importantly, we also found that, to incorporate in the decision any variation in reference gene expression that is not due to the treatments but inherent to the gene itself, it is important to keep the biological replicates separated when entering data in geNorm or NormFinder. We observed an increased expression stability of AT4G34270 when using data input averaged per treatment and organ, as compared to data input using expression levels of individual samples. This suggests that, at least in our experimental system, variability in AT4G34270 gene expression exists between different samples that is not due to the treatments. Also Czechowski et al. (2005) used an averaged data input (expression levels of cDNA pools) and identified AT4G34270 among the best reference genes.

In conclusion, we evaluated candidate reference genes for normalisation of gene expression in roots and leaves of Arabidopsis thaliana when studying the effect of increased but sub-lethal concentrations of $\mathrm{Cd}$ and $\mathrm{Cu}$. We also identified the same best three reference genes when considering $\mathrm{Cu}$ and $\mathrm{Cd}$ treatments separately, even though these metals have very different properties and effects towards the plants. Whereas $\mathrm{Cu}$ is redox-active and can directly induce oxidative stress, Cd is not redox-active and induces oxidative stress via indirect mechanisms (Chaoui and El Ferjani 2005; Romero-Puertas et al. 2004). We therefore anticipate that the three best reference genes are also suitable for use when studying the effects of increased exposure of Arabidopsis thaliana to other metals.

\section{Acknowledgements}

This study was funded by a Research Foundation-Flanders grant (project G.0436.06). Tony Remans is a post-doctoral research fellow of the Reseach Foundation-Flanders (FWO). Karen Smeets was supported by a PhD grant for from Hasselt University (tUl-impulsfinancieringtoxicology). We also thank Carine Put and Ann Wijgaerts for their technical assistance. 


\section{References}

Andersen CL, Jensen JL, Ørntoft TF (2004) Normalisation of real-time quantitative reverse transcription-PCR data: a model-based variance estimation approach to identify genes suited for normalisation, applied to bladder and colon cancer data sets. Cancer Res 64: 5245-5250

Baryla A, Carrier P, Franck F, Coulomb C, Sahut C, Havaux M (2001) Leaf chlorosis in oilseed rape plants (Brassica napus) grown on cadmium-polluted soil: causes and consequences for photosynthesis and growth. Planta 212: 696-709

Becher M, Talke IN, Krall L, Krämer U (2004) Cross-species microarray transcript profiling reveals high constitutive expression of metal homeostasis genes in shoots of the zinc hyperaccumulator Arabidopsis halleri. Plant J 37: 251-268

Bovet T, Eggmann M., Meylan-Bettex J, Polier P, Kammer E, Marin U, Feller E, Martinoia E (2003) Transcript levels of AtMRPs after cadmium treatment: induction of AtMRP3. Plant Cell Environ 26: 371-381

Brunner AM, Yakovlev IA, Strauss SH (2004) Validating internal controls for quantitative plant gene expression studies. BMC Plant Biol 4: 14

Cagnac O, Bourbouloux A, Chakrabarty D, Zhang MY, Delrot S (2004) AtOPT6 transports glutathione derivatives and is induced by primisulfuron. Plant Physiol 135: 1378-1387

Chaoui A, El Ferjani E (2005) Effects of cadmium and copper on antioxidant capacities, lignification and auxin degradation in leaves of pea (Pisum sativum L.) seedlings. Cr Biol 328: $23-31$

Cuypers A, Vangronsveld J, Clijsters H (2000) Biphasic effect of copper on the ascorbateglutathione pathway in primary leaves of Phaseolus vulgaris seedlings during the early stages of metal assimilation. Physiol Plant 110: 512-517

Cuypers A, Vangronsveld J, Clijsters H (2002) Peroxidases in roots and primary leaves of Phaseolus vulgaris copper and zinc phytotoxicity: a comparison. J Plant Physiol 159: 869-876 
Czechowski T, Stitt M, Altmann T, Udvardi MK, Scheible W-R (2005) Genome-wide identification and testing of superior reference genes for transcript normalisation in Arabidopsis. Plant Physiol 139: 5-17

Fargasova A (2001) Phytotoxic effects of Cd, Zn, Pb, Cu and Fe on Sinapis alba L. seedlings and their accumulation in roots and shoots. Biol Plant 44: 471-473

Hellemans J, Mortier G, De Paepe A, Speleman F, Vandesompele J (2007) qBase relative quantification framework and software for management and automated analysis of real-time quantitative PCR data.Genome Biol. 8(2): R19

Herbette S, Taconnat L, Hugouvieux V, Piette L, Magniette MLM, Cuine S, Auroy P, Richaud P, Forestier C, Bourguignon J, Renou J-P, Vavasseur A, Leonhardt N (2006) Genome-wide transcriptome profiling of the early cadmium response of Arabidopsis roots and shoots. Biochimie 88: 1751-1765

Jain M, Nijhawan A, Tyagi AK, Khurana JP (2006) Validation of housekeeping genes as internal control for studying gene expression in rice by quantitative real-time PCR. Biochem Biophys Res Comm 345: 646-651

Jonak C, Nakagami H, Hirt H (2004) Heavy metal stress. Activation of distinct mitogenactivated protein kinase pathways by copper and cadmium. Plant Physiol 136: 3276-3283

Maksymiec W, Krupa Z (2006) The effects of short-term exposition to Cd, excess Cu ions and jasmonate on oxidative stress appearing in Arabidopsis thaliana. Environ Exp Bot 57: 187-194

Mittler R, Vanderauwera S, Gollery M, Van Breusegem F (2004) Reactive oxygen gene network of plants. Trends Plant Sci 9: 490-498

Nicot N, Hausman JF, Hoffmann L, Evers D (2005) Housekeeping gene selection for realtime RT-PCR normalisation in potato during biotic and abiotic stress. J Exp Bot 56: 29072914 
Ortega-Villasante C, Rellan-Alvarez R, Del Campo FF, Carpena-Ruiz RO, Hernandez LE (2005) Cellular damage induced by cadmium and mercury in Medicago sativa. J Exp Bot 56: 2239-2251

Pandey N, Sharma CP (2002) Effect of heavy metals $\mathrm{Co}^{2+}, \mathrm{Ni}^{2+}$ and $\mathrm{Cd}^{2+}$ on growth and metabolism of cabbage. Plant Sci 163: 753-758

Pfaffl MW, Tichopad A, Prgomet C, Neuvians TP (2004) Determination of stable housekeeping genes, differentially regulated target genes and sample integrity: BestKeeper-Excel-based tool using pair-wise correlations. Biotechnol Lett 26: 509-15

Prasad SM, Zeeshan M (2005) UV-B radiation and cadmium induced changes in growth, photosynthesis, and antioxidant enzymes of cyanobacterium Plectonema boryanum. Biol Plant 49: 229-236

Ransbotyn V, Reusch TBH (2006) Housekeeping gene selection for quantitative real-time PCR assays in the seagrass Zostera marina subjected to heat stress. Limnology and Oceanography-methods 4: 367-373

Rodriguez-Serrano M, Romero-Puertas MC, Zabalza A, Corpas FJ, Gomez M, Del Rio LA, Sandalio LM (2006) Cadmium effect on oxidative metabolism of pea (Pisum sativum L.) roots. Imaging of reactive oxygen species and nitric oxide accumulation in vivo. Plant Cell Environ 29: 1532-1544

Romero-Puertas MC, Rodriguez-Serrano M, Corpas FJ, Gomez M, Del Rio LA, Sandalio LM (2004) Cadmium-induced subcellular accumulation of $\mathrm{O}_{2}{ }^{--}$and $\mathrm{H} 2 \mathrm{O} 2$ in pea leaves. Plant Cell Environ 27: 1122-1134

Smeets K, Cuypers A, Lambrechts A, Semane B, Hoet P, Van Laere A, Vangronsveld J (2005) Induction of oxidative stress and antioxidative mechanisms in Phaseolus vulgaris after Cd application. Plant Physiol Biochem 43: 437-444 
Smeets K, Ruytinx J, Van Belleghem F, Semane B, Lin D, Vangronsveld J, Cuypers A (2007) Critical evaluation and statistical validation of a hydroponic culture system for Arabidopsis thaliana. Plant Physiol Biochem: doi:10.1016/j.plaphy.2007.09.014

Sunkar R, Kapoor A, Zhu JK (2006) Posttranscriptional induction of two Cu/Zn superoxide dismutase genes in Arabidopsis is mediated by downregulation of miR398 and important for oxidative stress tolerance. Plant Cell 18: 2051-2065

Talke IN, Hanikenne M, Kramer U (2006) Zinc-dependent global transcriptional control, transcriptional deregulation, and higher gene copy number for genes in metal homeostasis of the hyperaccumulator Arabidopsis halleri. Plant Physiol 142: 148-167

Thellin O, Zorzi W, Lakaye B, De Borman B, Coumans B, Hennen G, Grisar T, Igout A, Heinen E (1999) Housekeeping genes as internal standards: use and limits. J Biotechnol 75: 291-295

Vandesompele J, De Preter K, Pattyn F, Poppe B, Van Roy N, De Paepe A, Speleman F (2002) Accurate normalisation of real-time quantitative RT-PCR data by geometric averaging of multiple internal control genes. Genome Biol 3(7): research0034.1-0034.11

Weber M, Trampczynska A, Clemens S (2006) Comparative transcriptome analysis of toxic metal responses in Arabidopsis thaliana and the $\mathrm{Cd}^{2+}$-hypertolerant facultative metallophyte Arabidopsis halleri. Plant Cell Environ 29: 950-963

Yeh CM, Chien PS, Huang HJ (2007) Distinct signalling pathways for induction of MAP kinase activities by cadmium and copper in rice roots. J Exp Bot 58: 659-671

\section{Figure legends}

Fig. 1a-d Evaluation of reference genes in Arabidopsis seedlings after exposure to relatively low and high concentrations of $\mathrm{Cd}$ or $\mathrm{Cu}$ during 24h. Stepwise exclusion of the reference genes with the highest M-value (lowest expression stability) resulted in ranking of the candidate reference genes when both $\mathrm{Cd}$ and $\mathrm{Cu}$ treatments were considered (a), and when $\mathrm{Cd}$ treatments (b) and $\mathrm{Cu}$ treatments (c) were considered separately. The pairwise variation 
measure " $\mathrm{Vn} / \mathrm{n}+1$ " measured the effect of adding additional reference genes on the normalisation factor for these treatments (d) 
Table 1 Reference genes and their primer sequences (from Czechowski et al. 2005) that were selected for evaluation of expression stability during $\mathrm{Cd}$ and $\mathrm{Cu}$ exposure

\begin{tabular}{|c|c|c|c|}
\hline AGI & Annotation & forward primer & reverse primer \\
\hline AT5G25760 & UBC & CTGCGACTCAGGGAATCTTCTAA & TTGTGCCATTGAATTGAACCC \\
\hline AT2G28390 & SAND family & AACTCTATGCAGCATTTGATCCACT & TGATTGCATATCTTTATCGCCATC \\
\hline AT2G32170 & expressed & ATCGAGCTAAGTTTGGAGGATGTAA & TCTCGATCACAAACCCAAAATG \\
\hline AT4G26410 & expressed & GAGCTGAAGTGGCTTCCATGAC & GGTCCGACATACCCATGATCC \\
\hline AT4G33380 & expressed & TTGAAAATTGGAGTACCGTACCAA & TCCCTCGTATACATCTGGCCA \\
\hline AT4G34270 & TIP41-like & GTGAAAACTGTTGGAGAGAAGCAA & TCAACTGGATACCCTTTCGCA \\
\hline AT5G08290 & mitosis protein YLS8 & TTACTGTTTCGGTTGTTCTCCATTT & $\begin{array}{l}\text { CACTGAATCATGTTCGAAGC } \\
\text { AAGT }\end{array}$ \\
\hline AT5G15710 & F-box protein & TTTCGGCTGAGAGGTTCGAGT & GATTCCAAGACGTAAAGCAGATCAA \\
\hline AT5G46630 & $\begin{array}{l}\text { clathrin adaptor } \\
\text { complex subunit }\end{array}$ & TCGATTGCTTGGTTTGGAAGAT & GCACTTAGCGTGGACTCTGTTTGATC \\
\hline AT5G55840 & PPR gene & AAGACAGTGAAGGTGCAACCTTACT & AGTTTTTGAGTTGTATTTGTCAGAGAAAG \\
\hline АТЗG18780 & ACT2 & CTTGCACCAAGCAGCATGAA & CCGATCCAGACACTGTACTTCCTT \\
\hline AT4G05320 & UBQ10 & GGCCTTGTATAATCCCTGATGAATAAG & AAAGAGATAACAGGAACGGAAACATAGT \\
\hline AT5G60390 & EF-1 $1 \alpha$ & TGAGCACGCTCTTCTTGCTTTCA & GGTGGTGGCATCCATCTTGTTACA \\
\hline
\end{tabular}


Table 2 Amplification efficiencies ( \pm SD) of the reference genes measured by a four-fold dilution series of one sample and average Ct values ( \pm SD) obtained by measuring expression in the biological replicates of the listed treatment / organ combination

\begin{tabular}{|c|c|c|c|c|c|c|c|c|c|c|c|c|c|c|c|c|c|c|c|c|c|c|c|c|c|c|c|c|c|c|}
\hline \multirow{3}{*}{$\begin{array}{c}\text { Efficiency > } \\
\text { Treatment / Organ }\end{array}$} & \multirow{2}{*}{\multicolumn{3}{|c|}{$\begin{array}{c}\text { AT2G28390 } \\
2.0661 \pm 0.0561\end{array}$}} & \multirow{2}{*}{\multicolumn{3}{|c|}{\begin{tabular}{|c|} 
AT4G26410 \\
$1.9054 \pm 0.0933$
\end{tabular}}} & \multicolumn{3}{|c|}{ AT4G34270 } & \multicolumn{3}{|c|}{ AT5G08290 } & \multicolumn{3}{|c|}{ AT5G15710 } & \multicolumn{3}{|c|}{ AT5G25760 } & \multicolumn{3}{|c|}{ AT5G55840 } & \multicolumn{3}{|c|}{ ACT2 } & \multicolumn{3}{|c|}{ EF-1a } & \multicolumn{3}{|c|}{ UBQ10 } \\
\hline & & & & & & & 2.086 & $7 \pm$ & 0.0298 & 2.0796 & $6 \pm 0$ & 0.0162 & $2.141 \varepsilon$ & $8 \pm$ & 0.0472 & 1.9395 & $5 \pm$ & 0.0478 & 1.905 & $6 \pm 0$ & 0.0382 & 1.9631 & $1 \pm$ & 0.0136 & 2.0478 & $8 \pm 0$ & 0.0326 & 2.0963 & $3 \pm$ & 0.0435 \\
\hline & $\mathrm{Ct}$ & \pm & Stdev & & \pm & Stdev & & \pm & Stdev & & $\pm s$ & Stdev & $\mathrm{Ct}$ & - & Stdev & $\mathrm{Ct}$ & \pm & Stdev & $\mathrm{Ct}$ & \pm & Stdev & $\mathrm{Ct}$ & \pm & Stdev & $\mathrm{Ct}$ & \pm & Stdev & $\mathrm{Ct}$ & - & Stdev \\
\hline I & 54 & \pm & 0.00 & .41 & \pm & 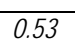 & 23.10 & \pm & 0.44 & 20.58 & \pm & 36 & \begin{tabular}{|l|}
26.42 \\
\end{tabular} & - & .51 & 23.18 & \pm & 0.52 & 28.90 & \pm & .08 & 22.65 & - & 0.56 & \begin{tabular}{|l|}
17.65 \\
\end{tabular} & \pm & .43 & 19.11 & + & .21 \\
\hline $2 \mu \mathrm{M}$ & 24.61 & \pm & 0.40 & 23.76 & \pm & 0.64 & 24.35 & \pm & 36 & .93 & \pm & 0.38 & 26.40 & 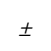 & 34 & .11 & \pm & 0.36 & 29.29 & \pm & 83 & 23.54 & \pm & 36 & 18.79 & \pm & 72 & 9.46 & 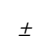 & 43 \\
\hline $10 r$ & 24.36 & \pm & 0.32 & 2 & \pm & 0.70 & 24.86 & \pm & 2.93 & 20.47 & \pm & 8 & 2 & \pm & 0.32 & 23.31 & \pm & 6 & 29.95 & \pm & & 6 & \pm & & 39 & \pm & & 67 & \pm & 37 \\
\hline $0.5 \mu \mathrm{M} \mathrm{Cu} /$ leaves & 24.53 & \pm & 26 & 24.45 & \pm & 0.30 & 23.82 & \pm & 0.67 & 20.67 & \pm & 0.63 & 2 & \pm & & & - & 77 & 3 & \pm & & 3 & \pm & & 7.98 & \pm & 29 & 13 & \pm & 77 \\
\hline $2 \mu \mathrm{M} \mathrm{Cu} /$ leaves & 24.14 & \pm & 0.15 & 23.18 & \pm & 0.61 & 23.08 & \pm & 0.55 & 20.32 & \pm & 0.06 & 25.78 & \pm & 0.24 & 23.54 & \pm & 0.88 & 28.78 & \pm & 0.30 & 22.95 & \pm & 0.24 & 18.00 & \pm & 0.37 & 18.41 & \pm & 0.11 \\
\hline control / roots & 23.59 & \pm & 0.19 & 22.65 & \pm & 0.91 & 23.17 & \pm & 1.48 & 19.54 & \pm & 0.23 & 25.59 & \pm & 0.32 & 23.09 & \pm & 1.35 & 30.76 & \pm & 1.85 & 20.89 & \pm & 0.41 & 16.91 & \pm & 0.35 & 18.05 & \pm & 0.28 \\
\hline $2 \mu \mathrm{M} \mathrm{Cd} /$ roots & 24.11 & \pm & 0.55 & 23.02 & \pm & 0.94 & 23.46 & \pm & 1.64 & 19.78 & \pm & 0.43 & 26.09 & \pm & 0.71 & 23.20 & \pm & 1.24 & 30.09 & \pm & 0.38 & 21.89 & \pm & 1.17 & 17.35 & \pm & 0.97 & 18.52 & \pm & 0.64 \\
\hline $10 \mu \mathrm{M} \mathrm{Cd} /$ roots & 23.34 & \pm & 0.39 & 22.40 & \pm & 0.75 & 22.57 & \pm & 1.58 & 19.33 & \pm & 0.08 & 25.53 & \pm & 0.63 & 22.69 & \pm & 0.46 & 29.59 & \pm & 0.33 & 20.88 & \pm & 0.21 & 16.93 & \pm & 0.81 & 17.92 & \pm & 0.40 \\
\hline $0.5 \mu \mathrm{M} \mathrm{Cu} /$ roots & 24.12 & \pm & 1.80 & 22.54 & \pm & 0.69 & 22.41 & \pm & 0.57 & 19.42 & \pm & 0.23 & 25.25 & \pm & 0.25 & 23.37 & \pm & 0.87 & 29.21 & \pm & 0.35 & 20.82 & \pm & 0.45 & 15.96 & \pm & 0.29 & 18.00 & \pm & 0.23 \\
\hline $2 \mu \mathrm{M} \mathrm{Cu} /$ roots & 24.68 & \pm & 0.85 & 23.94 & \pm & 0.60 & 25.56 & \pm & 3.33 & 20.51 & \pm & 0.75 & 26.60 & \pm & 0.70 & 23.96 & \pm & 1.66 & 30.15 & \pm & 1.27 & 23.26 & \pm & 1.25 & 18.10 & \pm & 1.45 & 17.90 & \pm & 0.57 \\
\hline
\end{tabular}



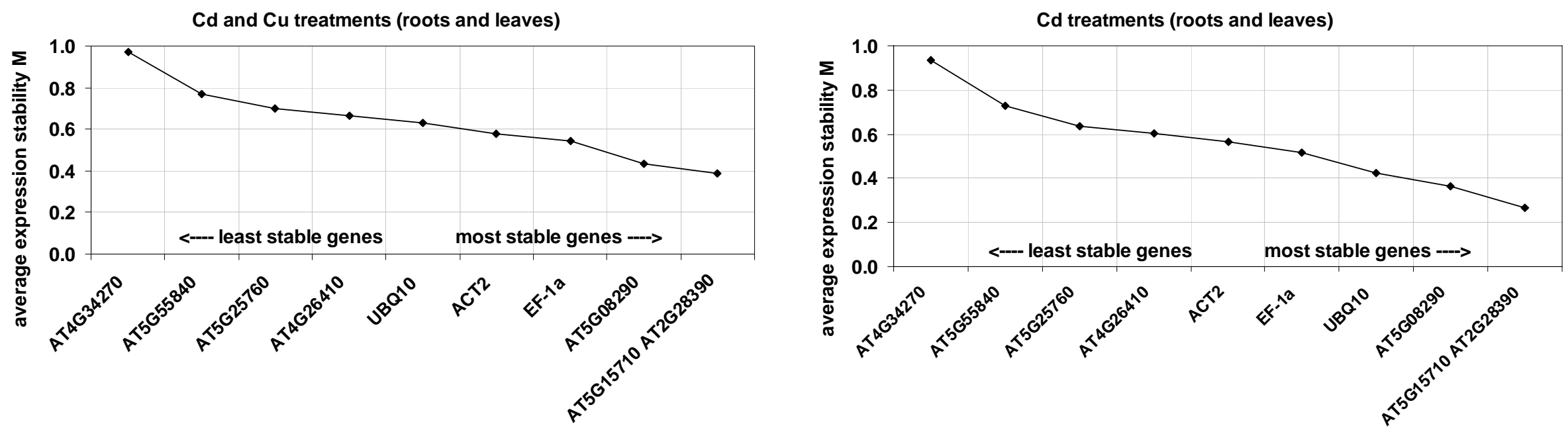

C

d
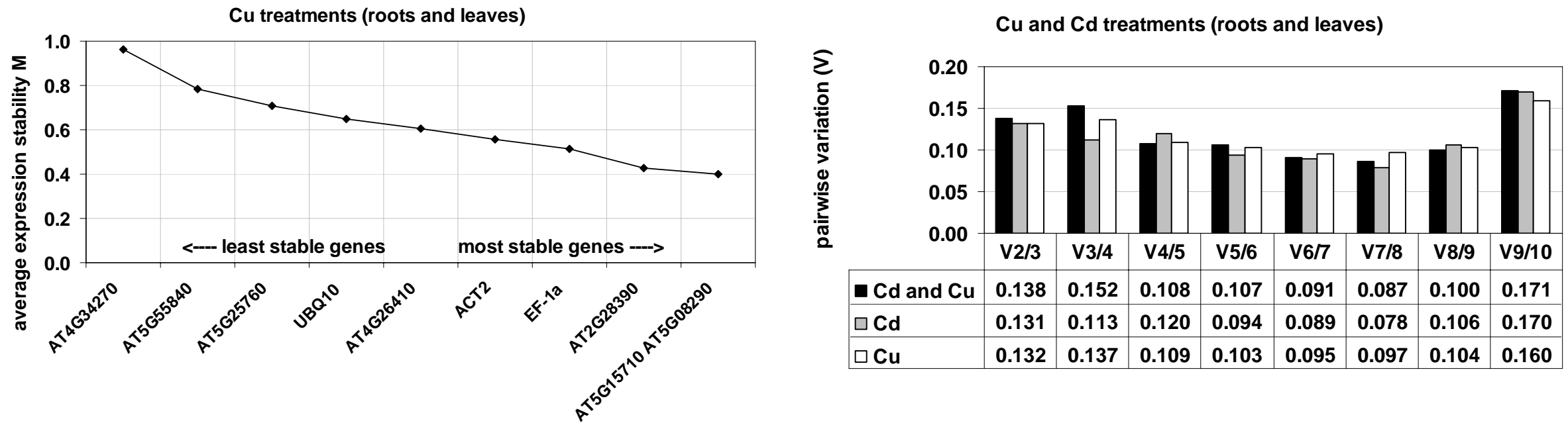
